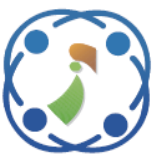

\title{
EMG Signals Classification of Wide Range Motion Signals for Prosthetic Hand Control
}

\author{
Noof T. Mahmood ${ }^{1 *}$ \\ Mahmuod H. Al-Muifraje ${ }^{2}$ \\ Sameer K. Salih ${ }^{3}$ \\ ${ }^{1}$ Electrical Engineering Department, University of Technology, Iraq \\ ${ }^{2}$ Department of Computer Techniques Engineering, Al-Rasheed University Collage, Iraq \\ ${ }^{3}$ Ministry of Sciences and Technology, Iraq \\ * Corresponding author's Email: noofthabit@gmail.com
}

\begin{abstract}
The recent revolution in the biomedical field carried out the researchers to work on the prosthetic technique because it reflects the amputee's need. Therefore, the electromyography (EMG) signals generated by muscle contractions are used to implement the prosthetic human body parts. This paper presents a pattern recognition system based on two EMG data; the first EMG data represents the general body movements collected from biceps and triceps muscles for six different motions, i.e., bowing, clapping, handshaking, hugging, jumping, and running. The Root Mean Square, Difference Absolute Standard Deviation Value, and Principal Component Analysis extract the raw signal data features and enhance classification accuracy. The machine learning method is applied, i.e., Support Vector Machine (SVM) and K-Nearest Neighbours (KNN) are used for data classification; the results show high accuracy reached 94.8\% and 98.9\%, respectively. Whereas, the second EMG data is selected to be more specific in hand movements, i.e., cylindrical, spherical, palmar, lateral, hook, and tip motions, because these significant motions are the first step implementing any prosthetic hand. Consequently, the mean, Standard Deviation Value, and Principal Component Analysis extract the raw signal feature. Meanwhile, the same algorithm used in the first data classification is also used to classify the second data because it shows high accuracy and good performance. SVM algorithm is used to classify the data and achieved high training accuracy, reaching $89 \%$. The high training accuracy for different hand movements is considered an essential step toward implementing human prosthetic parts to help the people who suffer from an amputee.
\end{abstract}

Keywords: EMG, SVM, KNN, Prosthetic.

\section{Introduction}

Physical therapy plays a significant role in the rehabilitation stage. So, many efforts are made to elucidate the leverage of medical/ clinical and human-machine interface (HMI) applications [1]. The dominant control methods are using electromyography (EMG) signals generated by muscle constriction to implement the prosthetic human body parts [2]. Therefore, robotic has the effectiveness to increase the independence of the individuals living lifestyle with their disabilities. The objective of this work is to improve the life quality that happens by empowering people to achieve a wide range of daily responsibilities within a little time. Humanoid robots can prepare robotic limbs to make the physical actions of individuals and the intelligent robotics industry. Nowadays, the most widely used are the robotic hands and arms. The robotic hands would have the ability to achieve the primary skills like grasp and transfer of objects from one place to another similar to non-amputees' individuals do. Therefore, depending on the object shapes, the human hand actions must be trained and applied to the designed robotics hand. The intuitive approach creates an interface that brings the activity of the muscle and records it using EMG sensors [3].

EMG signals are a way for estimating the electrical signal that relate to skeletal muscle; the electrical signal consists of some motor unit action potentials (MUAPs) [4]. The combination of muscle fibre action potentials from all the muscle fibres of a 
single motor unit is called the MUAP [5].

The pattern recognition (PR) system based on electromyography signals is divided into three main parts; data collection, feature extraction, and classification of the input data.

First stage, there are many methods to collect the electromyography signals from the muscles, first method by collecting data from the skin surface using different sensors such as Myo armband, which is considered a commercial way. The second method is collecting the data using a needle that is considered as a medical method and rarely used in research [6, 7].

The second stage is extracting the features. Feature extraction can be analysed in different domains (time or frequency or time-frequency) analysis to acquire significative information from EMG signals. The most common domain used in EMG signals is the time domain which is represented by mean absolute value (MAV), zero crossings (ZC), nth-order autoregressive (AR), mean, log detector, variance (VAR), wavelength (WL), root-meansquare value (RMS), simple square integral (SSI), the sign of slope changes (SS), modified mean absolute value (MMAV), EMG integral (IEMG), average amplitude change (AAC), histogram, Willison amplitude and sample entropy [8].

The final stage in PR is data classification to specify to which class the extracted features fit. The most common machine learning classifiers are Support Vector Machine (SVM), decision tree, KNearest Neighbour, Hidden Markov (HM), and Linear discriminant analysis (LDA) [9]. Currently, the prosthetic technique is an artificial part of resolving the problem of amputees' parts. The appearance of the consumer-level 3D printers supports the prosthetics implementation. Thus, the develop prosthetics has gained popularity over traditional prosthetics, which are expensive and unaffordable to many users [10]. The main challenge in the mechanical standpoint is how to mix many degrees of freedom (DOF) [11]. As a result, increment in DOF is caused by decreasing in the gripping force, which leads to an unstable system. Also, increasing DOFs will lead to a rise in actuators number and high failure probability so that the prosthetic hand will need high maintenance and difficult manufacture [12].

The main problem is summarized by implementing prosthetic hand for people with amputees to perform hand movements. Hence, the EMG signals have to collect from different muscles then classifies according to the movement type. After that, the output of the classifiers will be entered into the microcontroller to control the prosthetic part.
Therefore, this paper firstly works on the EMG signals classification for general body movements (bowing, clapping, handshaking, hugging, jumping, and running). The work passed to the main contribution by classifying the basic hand movements (cylindrical, spherical, palmar, lateral, hook, and tip) to achieve high training accuracy. The novelty of this work by achieving high classification accuracy on the EMG signals for fingers movement; because these signals are hard to detect in the classification; due to the similarity between the finger's signals, low amplitude, and high noise that the signals suffer from it.

This paper coordinated as follows; the 2nd section describes the methodology of this work, starting from the data collection, features extraction, and learning algorithms. The 3rd section clarifies the obtained results. In contrast, the 4th section discusses the results and makes a comparison with other works. Finally, the 5th section offers the conclusion of the work; meanwhile, the last section proposed the future step for this work.

\section{Methodology}

This work aims to perform a step forward toward designing a prosthetic hand that can move each finger independently. Hence, the first step is achieved by working on the general body movements, which are considered a broad muscles active signal, and tried to obtain high training accuracy. While in the second step, the work will pass to another part of actions which are the basic hand movements; this part can be closer to the aim that stats on moving each finger independently.

The work is started by designing a system for pattern recognition (PR) of the EMG signal. Fig. 1 shows the proposed algorithm for pattern recognition system; (1-A) the general body movements (bowing, clapping, handshaking, hugging, jumping, and running) used three features and two classifiers. While, figure (1-B) the basic hand movements (cylindrical, spherical, palmar, lateral, hook, and tip) used anther three feature and support vector machine as a classifier. The first step for this work is collecting the data since the EMG signals differ from one person to others; the authors work on collecting EMG data from more than six participants of different gender and different ages by using Myo armband sensor. The second step is to simplify the raw collecting EMG signals by extracting time-domain features to prepare the extracted signals for the classifications stage. The final step will be choosing suitable classifiers from the machine learning algorithms groups to classify the EMG signals for different movements. 


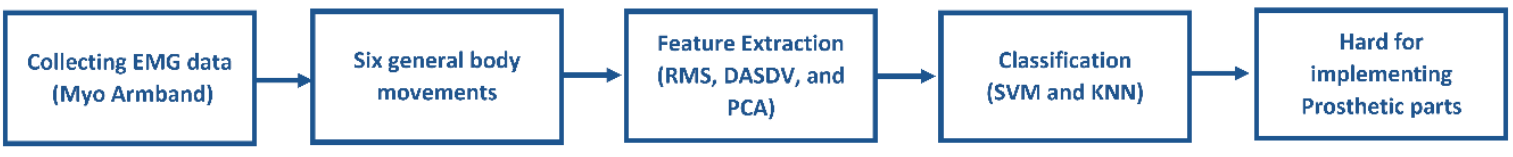

B

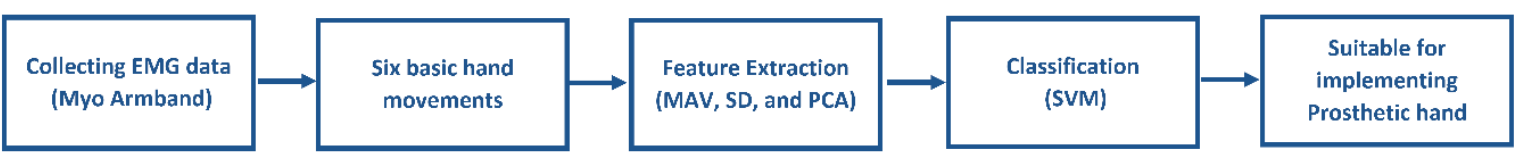

Figure. 1 The proposed algorithm for pattern recognition system: (A) general body movements, (B) the basic hand movements

\subsection{Data collection}

The EMG signal data is collected from different subjects using Myo armband which contains eight EMG sensors distributed in $360^{\circ}$. Then transferred to the PC using Bluetooth. In this work, two types of EMG signals are collected:

- $\quad$ Recording six general body movements, i.e., bowing, clapping, handshaking, hugging, jumping, and running) for two males and one female. eight sensors of the Myo armband are corresponding to eight inputs in time domain and the amplitude of the

signal represents the voltage of the required muscles, and each time series consists of 9000 samples. The eight sensors cover the following body part; right arm, left arm, right leg, and left leg, taking two muscles; the biceps and the triceps. The amplitude of the EMG signals represents muscle construction for high voltage and muscle relaxation for low voltage. Some of the obtained general body movements have a high amplitude, i.e., jumping, Running and hugging due to the strong muscles' contractions.

- Recording six basic hand movements, i.e., (cylindrical, spherical, hook, palmar, tip, and lateral) for two males and three females. Eight sensors of the Myo armband are corresponding to eight inputs in time domain and the amplitude of the signal represents the voltage of the required muscles, and each time series consists of 3000 samples, the eight sensors cover the elbow. As a notice, Fig. 2 shows the raw EMG signals for basic hand movements; the amplitude of the EMG signals represents muscle contraction for high voltage and muscle relaxation for low voltage. In this part, the obtained EMG signals have low amplitude due to the low tension on muscles responsible for finger movements.

After collecting EMG signals for the two types (general body movements and basic hand movements), the raw signals have to be simplified by filtering or extracting features before classifying the signals. The filtering step is cancelled in this work due to the Myo Armband having a low additive noise. Therefore, the time-domain analysis is directly used to extract the features from the raw EMG signals.

\subsection{Feature extraction}

The benefit the feature extraction is to avoid extensive data and time consuming for signal processing. Therefore, many feature extraction types are used to reduce the raw data dimensions and produce new vectors that will enter the classification stage instead of the raw data. So, the new vectors must contain all the required information to obtain fast training $[12,13]$.

In this work, the EMG signals are analysed in offline mode using MATLAB 2019a. The recoded signals are then segmented in a window size of 200 $\mathrm{ms}$ and an increment of $150 \mathrm{~ms}$. The window is analysed using Root Mean Square (RMS), Difference Absolute Standard Deviation Value (DASDV), Mean Absolute Value (MAV), Standard deviation (SD) and Principal Component Analysis (PCA) is used for dimension reduction because of reduction in time and space complexities. The dimensions of the new components will be uncorrelated and orthogonal to each other $[14,15]$. The following equations used for feature extractions [16-20]:

$$
\begin{aligned}
& R M S=\sqrt{\frac{1}{N} \sum_{i=1}^{N} X i^{2}} \\
& D A S D V=\sqrt{\frac{1}{N-1} \sum_{i=1}^{N-1}\left(X_{i+1}+X_{i}\right)^{2}} \\
& M A V=\frac{1}{N} \sum_{n=1}^{N}\left|x_{n}\right| \\
& S D=\sqrt{1 /{ }_{N} \sum_{n=1}^{N}\left(x_{n}-M A V\right)^{2}}
\end{aligned}
$$



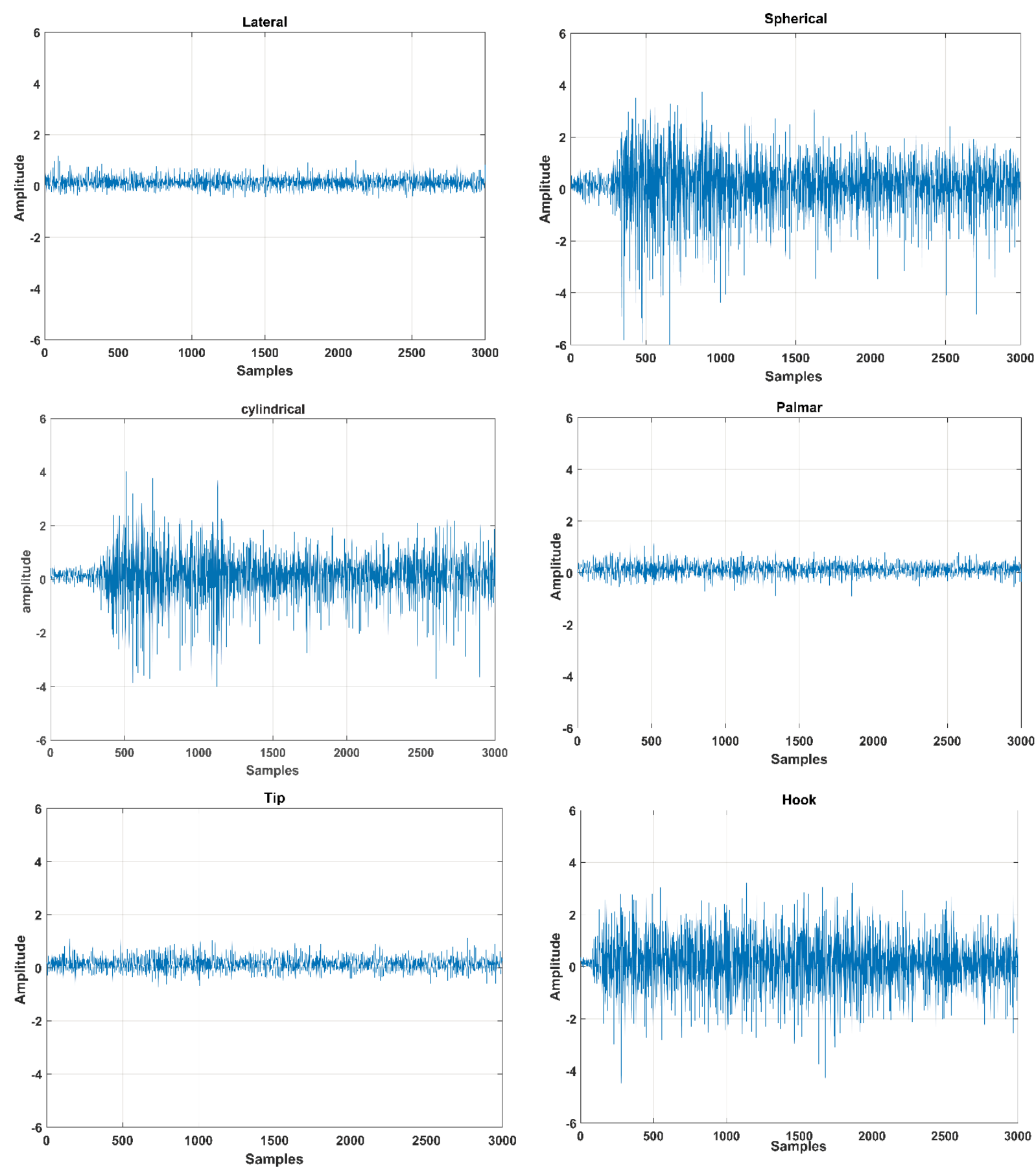

Figure. 2 The raw EMG signals for basic hand movements

Where in all equations above; $x$ : input EMG signal, $\mathrm{N}$ : the length of the input signals.

\subsection{Classification method}

After that, the Principal Component Analysis (PCA) is used for dimension reduction of the EMG signals because of the reduction in time and space complexities. after extracting the features from the raw data, the learning algorithm is chosen to classify the data according to the required categories [12].
This work uses the new features to train the model and assess the model performance using the (Matlab2019a). Then, machine learning is used to classify the types of EMG signals; machine learning contains multi- algorithms. In this work, the searchers apply all the machine learning algorithms; then choose the Support Vector Machine and K- Nearest Neighbours because both of them achieve high training accuracy: 


\subsubsection{Support vector machine (SVM)}

SVM is a powerful supervised machine learning technique that is used for regression or classification issues. The SVM finds the optimum separating hyperplane in data classification by building hyperplane or hyperplane groups with infinite dimensionality space [21].

The SVM can deal with linearly separable data and not linearly separable. Since this work deals with not linearly separable because it maps the data to a higher dimension and uses the radial basis function (RBF) to obtain a high classification rate [26, 27]. Therefore, the RBF is considered as the kernel function to generate non-linear classifiers. The cubic SVM is a type of SVM, and the equation of cubic SVM can be given as Eq. (5):

$k\left(x_{i}, x_{j}\right)=\left(x_{i}^{T}, x_{j}\right)^{3}$

The cubic SVM is preferred because the short time required form training in this work training time ranges from $3 \mathrm{sec}$ to $12 \mathrm{sec}$.

\subsubsection{K-nearest-neighbour's (KNN)}

KNN is supervised learning method and has a useful and accurate classification [22]. In the KNN method, the data represented in the vector space. The $\mathrm{k}$ represents a variable and selects as an essential factor, and $\mathrm{h}$ represents the least distance from the selected point if $\mathrm{k}$ is equal to $\mathrm{h}$, which means unselected point. In this work, the Euclidean distance is used to measure the distance between points using the given Eq. (6) [23, 24]:

$D_{i}=\sum_{k=1}^{h} \sqrt{\left(x_{i}-x_{k}\right)^{2}+\left(y_{i}-y_{k}\right)^{2}}$

Fine KNN Classifier is type of KNN that makes it finely itemized to distinguish among different classes with the number of neighbours set to $1[25,26]$.

\section{Results}

In this paper, to assess the usability of the proposed pattern recognition system, a series of experiments has been done. Actually, eight healthy participants (four males and four females) took part in experiments; here, the participants indicated as subjects. The experimental sessions are composed of two phases due to the different types of performed movements. The first one belongs to the training session of general body movements. The second phase contains the training session for the basic hand movements. The result of two experimental sessions as follows:
- General body movements: In this session, three participants ( 2 males, 1 female) have been taken to collect the raw EMG signals by applying the RMS, DASDV, and PCA to extract the features. After that, the SVM and fine KNN algorithms are used to classify six general body movements. The performance of the classifier model is described using the confusion matrix. This matrix clarifies the relationship between the predictive and the actual events.

The accuracy of the confusion matrix can determine from the following equation [27]:

$$
\text { Accuracy }=\frac{T N+T P}{T N+T P+F N+F P}
$$

Table 2 shows the three different experiments; each experiment has a different number of subjects. All three features are added together and applied in all experiments. The results also show how an increment in the number of subjects with an acceptable limit can positively affect the training accuracy percentage as noticed, the SVM is slower than the KNN in this session but still considered fast in training by taking a maximum of $99.41 \mathrm{sec}$. Fig. 3 shows the confusion matrix of the two classifiers (SVM and KNN) and obviously the KNN has higher

Table 1. Confusion matrix model

\begin{tabular}{|c|c|c|}
\hline $\begin{array}{c}\text { Confusion } \\
\text { Matrix }\end{array}$ & Predicted No & Predicted Yes \\
\hline Actual No & TN (True & FN (False \\
& Negative) & Negative) \\
\hline Actual Yes & FP (False & TP (True \\
& Negative) & Positive) \\
\hline
\end{tabular}

Table 2. Three different experiments for general movements

\begin{tabular}{|c|c|c|c|c|c|}
\hline $\begin{array}{l}\text { General } \\
\text { moveme } \\
\text { nts }\end{array}$ & $\begin{array}{l}\text { No. } \\
\text { of } \\
\text { subje } \\
\text { cts }\end{array}$ & $\begin{array}{l}\text { Featu } \\
\text { res }\end{array}$ & $\begin{array}{l}\text { Classif } \\
\text { ier }\end{array}$ & $\begin{array}{l}\begin{array}{l}\text { Ti } \\
\text { me } \\
(\mathrm{sec}\end{array} \\
\end{array}$ & $\begin{array}{l}\text { Accur } \\
\text { acy }\end{array}$ \\
\hline Exp. 1 & \multirow{2}{*}{1} & \multirow{6}{*}{$\begin{array}{c}\text { DASD } \\
\text { V, } \\
\text { RMS, } \\
\text { and } \\
\text { PCA }\end{array}$} & SVM & $\begin{array}{l}99 . \\
41\end{array}$ & $67.5 \%$, \\
\hline Exp. 1 & & & KNN & $\begin{array}{l}2.6 \\
3\end{array}$ & $87.9 \%$ \\
\hline Exp. 2 & \multirow{2}{*}{2} & & SVM & $\begin{array}{l}48 . \\
93\end{array}$ & $92.1 \%$ \\
\hline Exp. 2 & & & KNN & $\begin{array}{l}3.0 \\
4\end{array}$ & $98.3 \%$ \\
\hline Exp. 3 & \multirow{2}{*}{3} & & SVM & $\begin{array}{l}45 . \\
54\end{array}$ & $94.8 \%$ \\
\hline Exp.3 & & & KNN & $\begin{array}{l}4.4 \\
2\end{array}$ & $98.9 \%$ \\
\hline
\end{tabular}


Received: May 28, 2021. Revised: July 19, 2021.

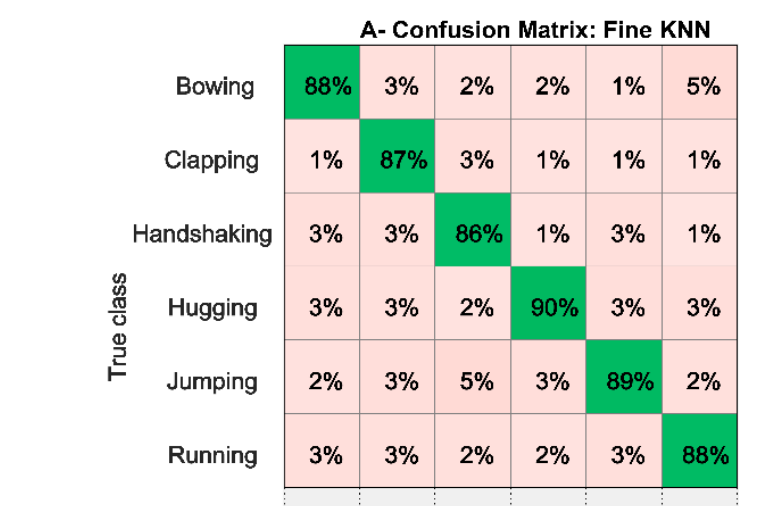

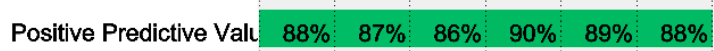

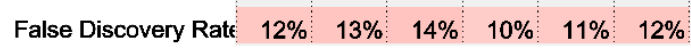

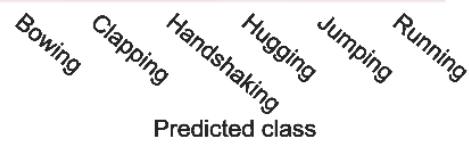

Predicted class
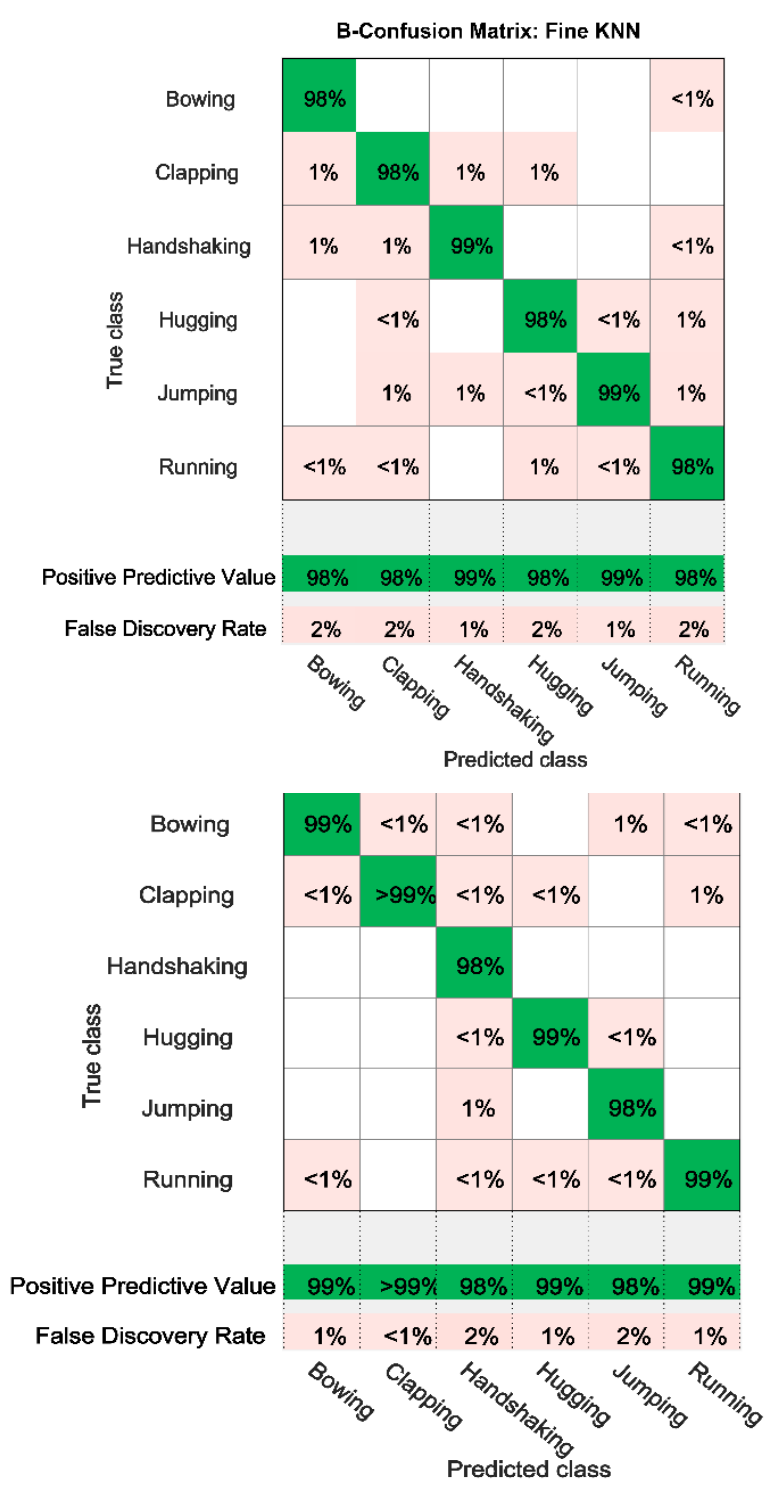
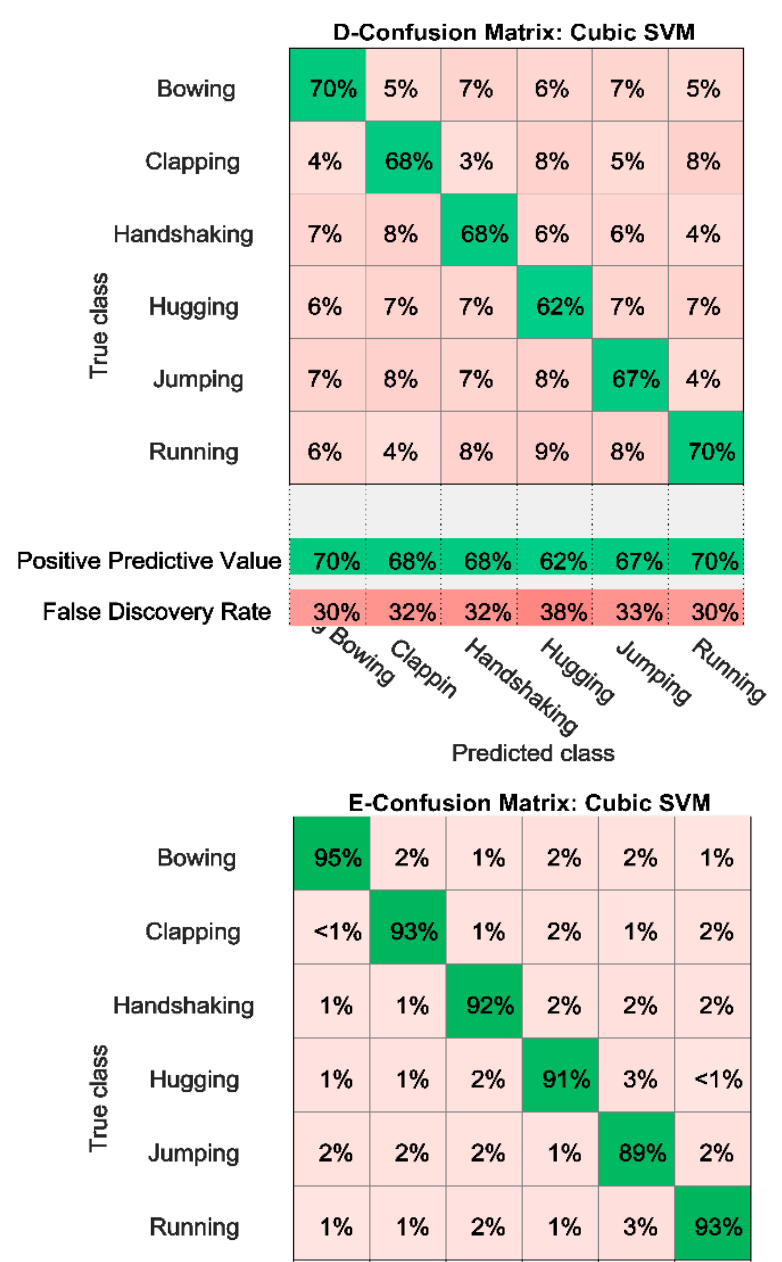

Positive Predictive Value

False Discovery Rate

\begin{tabular}{l|l|l|l|l|l}
$95 \%$ & $93 \%$ & $92 \%$ & $91 \%$ & $89 \%$ & $93 \%$
\end{tabular}

\begin{tabular}{l|l|l|l|l|l|}
$5 \%$ & $7 \%$ & $8 \%$ & $9 \%$ & $11 \%$ & $7 \%$ \\
\hline
\end{tabular}

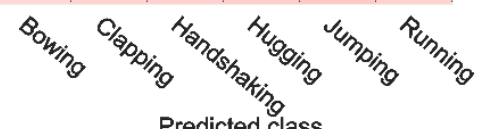

Predicted class

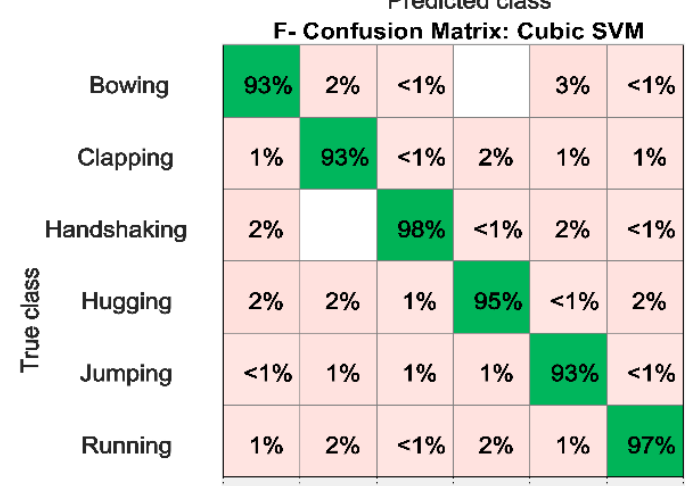

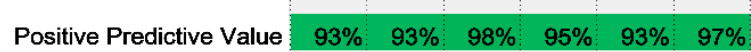

False Discovery Rate

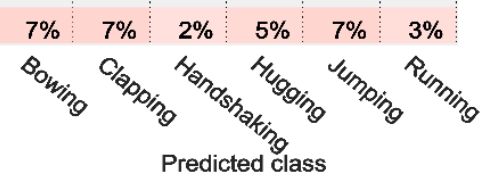

Figure. 3 Confusion matrix for; A, B, and C for Fine KNN for 1, 2, 3 subjects, respectively. E, D, and F for SVM for 1, 2, 3 subjects, respectively 
accuracy than the SVM in all experiments by accuracy reaching $98.9 \%$.

- $\quad$ Basic hand movements: In this session, five participants (two males, three females) have been taken to collect the raw EMG signals by applying the MAV, SD, and PCA to extract the features. After that, the SVM and algorithm are used to classify six basic hand movements. Table 3 shows the three different experiments; all the five subjects are added together and applied in all experiments. Each experiment has different numbers of features. In the first experiment, only the MAV is used to extract the feature; for this reason, the training accuracy reaches $57.4 \%$. While in the second one, both MAV and SD are applied together, so the training accuracy increases. In the last experiment, the MAV, SD, and PCA are used, and they achieve the highest training accuracy. As concluded, the increment in features with an acceptable limit affects increasing the accuracy percentage.

Fig. 4 shows the confusion matrices of SVM for the three experiments with different number of features and clarifies in details the accuracy percentage for each hand movements.

The scattering plot is applied in the second session for the third experiment to observe the relationship between the variables using different visual representations; each point represents an individual part. Where the dot represents the correct prediction, and $\mathrm{x}$ represents the incorrect prediction. Fig. 5 shows the scattering plot for three experiments using cubic SVM. So, the X-axis represents the MAV value for the first subject, and the $\mathrm{Y}$-axis represents the MAV value for the second subject in all three experiments.

In this work, the ROC (Receiver Operating Curve) analysis is applied in the second session for all the experiments due to the great attention in ML

Table 3. Three different experiments for basic hand

\begin{tabular}{|c|c|c|c|c|c|}
\hline $\begin{array}{l}\text { Basic } \\
\text { hand } \\
\text { moveme } \\
\text { nts }\end{array}$ & $\begin{array}{l}\text { No. of } \\
\text { subje } \\
\text { cts }\end{array}$ & $\begin{array}{l}\text { Featu } \\
\text { res }\end{array}$ & $\begin{array}{l}\text { Classif } \\
\text { ier }\end{array}$ & $\begin{array}{l}\begin{array}{l}\text { Ti } \\
\text { me } \\
(\text { sec }\end{array} \\
\end{array}$ & $\begin{array}{l}\text { Accur } \\
\text { acy }\end{array}$ \\
\hline Exp. 1 & \multirow{3}{*}{5} & MAV & \multirow{3}{*}{ SVM } & $\begin{array}{l}11 . \\
91\end{array}$ & $57.4 \%$ \\
\hline Exp. 2 & & $\begin{array}{l}\text { MAV } \\
\text { and } \\
\text { SD }\end{array}$ & & $\begin{array}{l}4.3 \\
5\end{array}$ & $87 \%$ \\
\hline Exp. 3 & & $\begin{array}{l}\text { MAV, } \\
\text { SD, } \\
\text { and } \\
\text { PCA }\end{array}$ & & $\begin{array}{l}6.3 \\
9\end{array}$ & $89 \%$ \\
\hline
\end{tabular}

to indicate the performance of the classifier. When the area under the curve (AUC) is considered the perfect ranking if it is equal to one. the proposed model can distinguish the correct classes. Fig. 6 shows the ROC for the three experiments. As noticed in the first figure, the classifier AUC is not good when it depends on one feature only. Meanwhile, in the second and the third figures, the number of features increase do the AUC has become perfect.

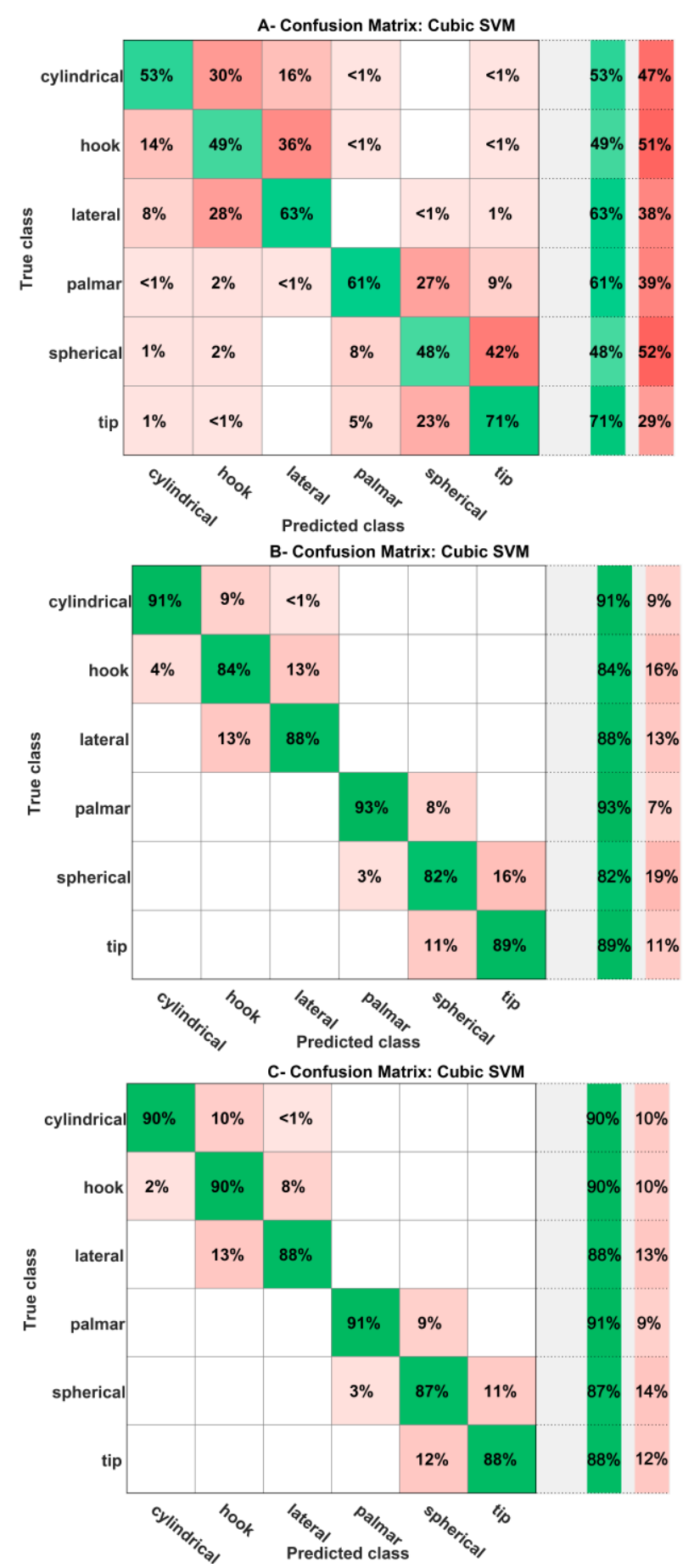

Figure. 4 The confusion matrix of SVM: (a) one feature, (b) two features, and (c) three features 


\section{Discussion}

Myo Armband is used in this work instead of the traditional sensors to solve noise issues and avoid the DC component in the raw signals, there is no need to
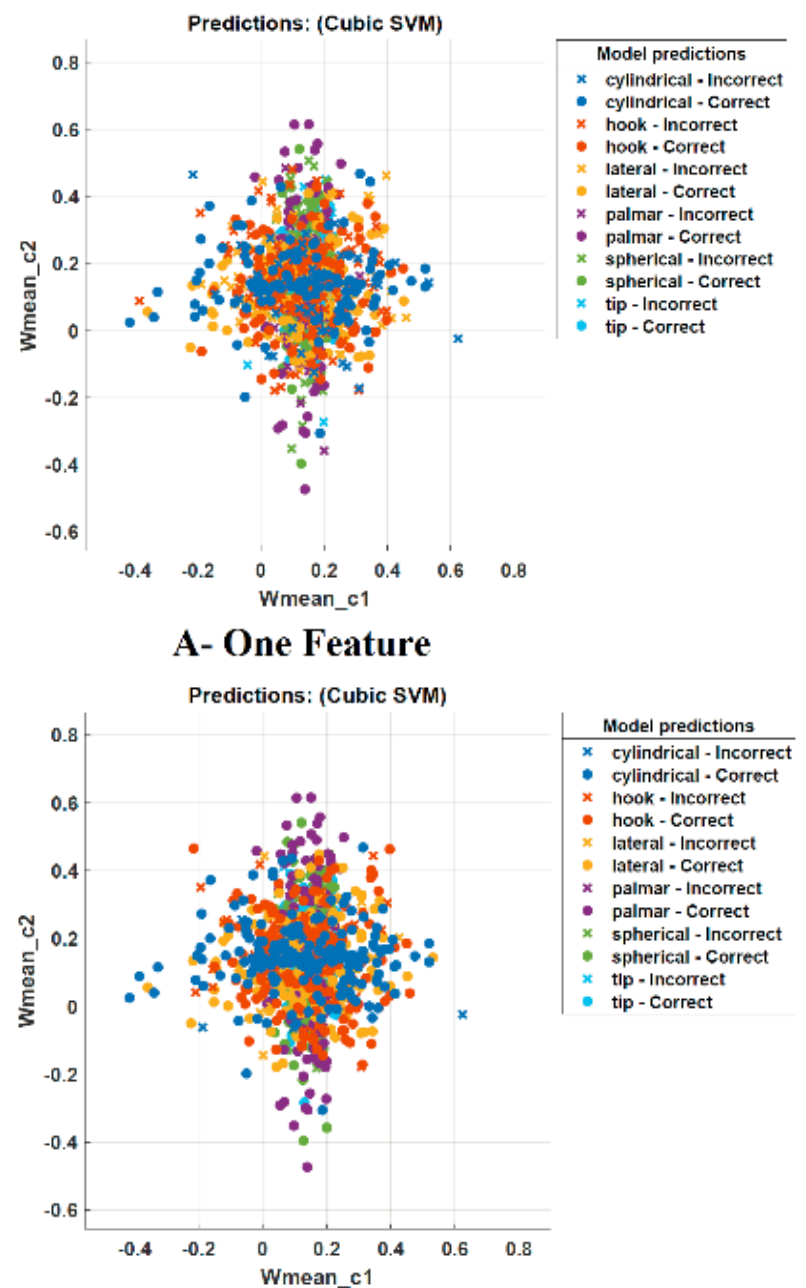

B- Two Features

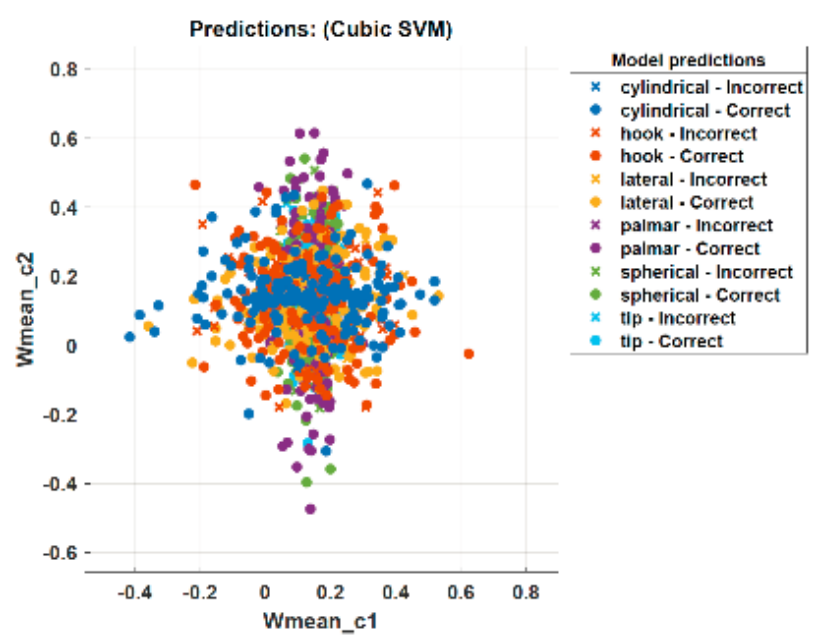

C- Three Features

Figure. 5 The scattering plot for three subjects using cubic SVM: (a) one features, (b) two features, and (c) three features use any filter. The data in each session was collected
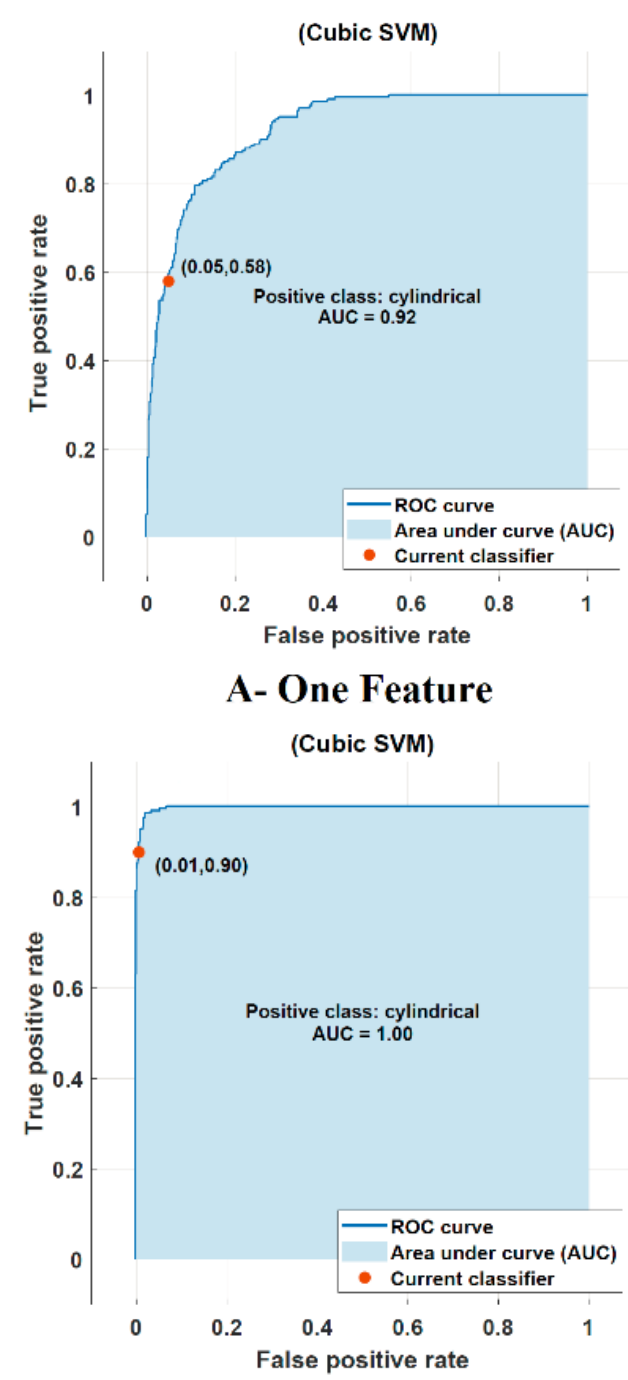

B- Two Features

(Cubic SVM)

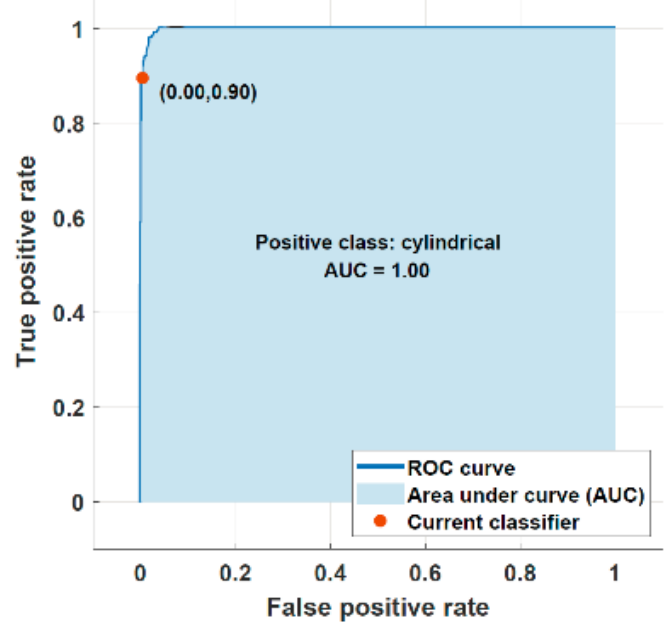

C- Three Features

Figure. 6 The ROC for three subjects: (a) one features, (b) two features, and (c) three features 


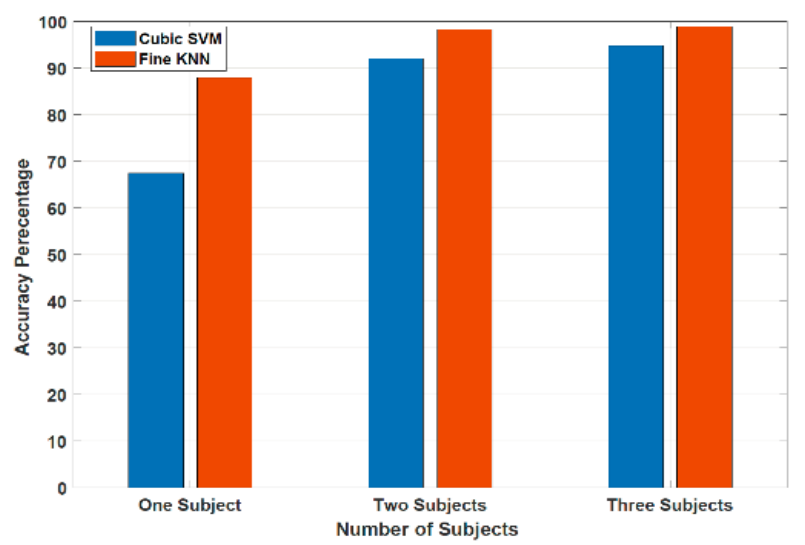

Figure. 7 The classification performance using different numbers of subjects

for six EMG movements from eight subjects. The time-domain analysis is used instead of frequency domain analysis to extract the raw EMG signals' features because it shows better performance in classifying the EMG signals.

In the general movements data, the SVM and KNN and shows fast training time; the average training time reaches $33.9 \mathrm{sec}$, and both algorithms achieved high accuracy.

In the general movements data, as noticed from all the three experiments, the first experiment has the lowest accuracies in all classifiers due to the small raw data. The results achieve the highest accuracies when the data of the three subjects used. This is due to the fact; the increased number of trained subjects will increase in data that will be trained and will increase accuracy. Fig. 7 shows the classification performance using different numbers of subjects.

According to the satisfying results in general body signals, the next aim was to achieve high classification accuracy for more specific movements (basic hand), by following the same previous procedure used for the general hand movements. The reason for choosing the basic hand movements because it considers the essential step toward implementing the prosthetic hand by covering the following motions (cylindrical, spherical, hook, palmar, tip, and lateral).

In the basic hand movements, the SVM was used and achieved acceptable training accuracy. Also, show fast average training time reaching $7.55 \mathrm{sec}$.

However, the obtained classification results in both parts show that the cubic support vector machine achieves the highest training accuracy among other SVM types due to the multi- hyper lines in this algorithm.

In the second session, three experiments are done, as noticed from the results; the first experiment has

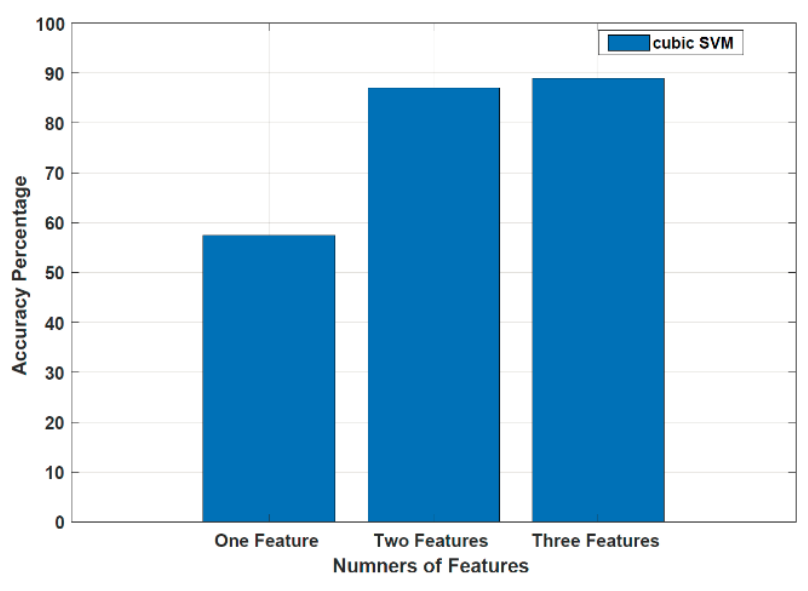

Figure. 8 The classification performance using different features

the lowest accuracy of $57.4 \%$ among all the experiments due to using only one feature (Mean). While, the results achieve the highest accuracy reaching $89 \%$ when using three features (Mean, standard deviation, and the principal component analysis). Therefore, the increasing number of features means increasing training accuracy because features extraction helps to analytic signals. Fig. 8 shows the classification performance using different features.

The final obtains training accuracy is $89 \%$; this accuracy percentage considered high because the selected hand movements are very similar to each other with a quit difference. Therefore, these motions can be divided into two groups according to the similarity of the movements; the first group contains cylindrical, spherical, and hook; the second group contains palmar, lateral, and tip.

The results of this work have been compared with five previous works as shown in Table 4. In general movements, the first study took only RMS and the SVM and achieved good accuracy. The second work chose the RMS, and Integrated Absolute Value (IAV) as features also select the KNN and Native Bayesian Pattern (NBP) as a classifier, and the authors achieved accuracy lower than the accuracy archived in this work. The third study used more than three features and used the linear regression and SVM as a classifier and achieved lower accuracy than this work achieved.

In the part of basic hand movements. Forth study took seven features and two classifiers (SVM and KNN) to achieve $63 \%$ accuracy using SVM. The fifth work chose thirteen features and a simple artificial neural network (ANN) to achieve $85.7 \%$ accuracy. Therefore, this work achieves the highest accuracy and fewer features among the previous works. 
Table 4. Compare this work with pervious works

\begin{tabular}{|c|c|c|c|c|}
\hline Ref. Year & Features & Movements & Classifier & Accuracy \\
\hline \multicolumn{5}{|c|}{ Part 1: General Movements } \\
\hline [28]. 2014 & RMS & $\begin{array}{l}\text { biceps brachii, quadriceps } \\
\text { femoris, tibialis anterior. }\end{array}$ & SVM & $95.75 \%$ \\
\hline [12]. 2018 & RMS and IAV & $\begin{array}{l}\text { Hand grasp } \\
\text { (Opposition) and release (Re- } \\
\text { position) }\end{array}$ & $\begin{array}{l}\text { KNN and } \\
\text { NBP }\end{array}$ & $92 \%, 94 \%$. \\
\hline [9]. 2019 & $\begin{array}{l}\text { mean, Kurtosis, Peak to Peak, } \\
\text { Shape Factor, energy, jitter, ZC, } \\
\text { and Spectral Spread. }\end{array}$ & Healthy and patients & $\begin{array}{l}\text { LR and } \\
\text { SVM }\end{array}$ & $\begin{array}{l}\text { SVM of } 94.1 \% \\
\text { LR of } \\
95.1 \% \\
\end{array}$ \\
\hline $\begin{array}{l}\text { This work. } \\
2021\end{array}$ & RMS, DASDV, and PCA & $\begin{array}{l}\text { Left Hand Clip, Left Hand } \\
\text { Down, Left Hand Up, Right } \\
\text { Hand Clip, Right Hand Down, } \\
\text { Right Hand Up. }\end{array}$ & $\begin{array}{l}\text { KNN and } \\
\text { SVM }\end{array}$ & $\begin{array}{c}\text { SVM of } 94.8 \% \text {, } \\
\text { and Fine KNN of } \\
98.9 \% .\end{array}$ \\
\hline \multicolumn{5}{|c|}{ Part 2: Basic Hand Movements } \\
\hline [29]. 2017 & $\begin{array}{l}\text { Mean Absolute Value (MAV), } \\
\text { Root Mean Square (RMS), } \\
\text { Simple Square Integral (SSI), } \\
\text { Waveform Length (WL), } \\
\text { Average Amplitude Change } \\
\text { (AAC), Difference Absolute } \\
\text { Standard Deviation Value } \\
\text { (DASDV), Log Detector (LOG) }\end{array}$ & $\begin{array}{l}\text { cylindrical, spherical, palmar, } \\
\text { lateral, hook, and tip }\end{array}$ & $\begin{array}{l}\text { Support } \\
\text { Vector } \\
\text { Machine } \\
\text { (SVM) } \\
\text { And k- } \\
\text { nearest } \\
\text { neighbor } \\
\text { (KNN) }\end{array}$ & $\begin{array}{l}\text { Best accuracy for } \\
\text { DASDV feature } \\
\text { for SVM of } 63 \%\end{array}$ \\
\hline [17]. 2016 & $\begin{array}{l}\text { (IEMG), (MAV), } \\
\text { (MAV1),(MAV2), } \\
\text { (VAR),(RMS),(WL), (SSI), } \\
\text { (DASDV),(AR), Hjorth } 1 \\
\text { (Activity), Hjorth } 2 \\
\text { (Mobility),Hjorth } 3 \\
\text { (Complexity) }\end{array}$ & $\begin{array}{l}\text { Hand Close, Hand Open. } \\
\text { Power Grip, Tripod. Pinch, } \\
\text { Hand force, Mouse, } \\
\text { Keyboard. }\end{array}$ & $\begin{array}{l}\text { artificial } \\
\text { neural } \\
\text { network } \\
(\mathrm{ANN})\end{array}$ & $85.70 \%$ \\
\hline $\begin{array}{l}\text { This work, } \\
2021\end{array}$ & Mean, STD, and PCA & $\begin{array}{l}\text { cylindrical, spherical, palmar, } \\
\text { lateral, hook, and tip }\end{array}$ & SVM & $89 \%$ \\
\hline
\end{tabular}

\section{Conclusion}

In this work, the proposed classification technique using machine learning (ML) shows a high training speed with two types of EMG data. The first part of this paper works on general movements; the number of the subjects (i.e., number of inputs to classifier) significantly increases accuracy. Therefore, the third experiment that uses three subjects shows the highest accuracy among other experiments. As noticed from the result, both Fine KNN and SVM achieve high accuracy, reaching $98.9 \%$ and $94.8 \%$, respectively.

The general movements were not enough to implement a specific prosthetic part. Therefore, the second part of this paper works on the basic hand movements; increasing the number of features significantly increases accuracy. Therefore, the third experiment that uses three subjects shows the highest accuracy among other experiments. As noticed from the result, the cubic SVM achieves the highest accuracy, reaching $89 \%$. Therefore, the high training accuracy of this work is useful to implement a genuine prosthetic hand based on EMG signals.

The next aim will be designing and implementing the EMG signals controller to be responsible for moving the hand fingers independently. That's mean taking the software part of this work to be converted to hardware.

\section{Conflicts of interest}

We confirm that there is no conflict of interest in this work

\section{Author contributions}

"Conceptualization, and Methodology, Noof T. Mahmood, Software; Noof T. Mahmood; Validation, Noof T. Mahmood; Formal analysis Noof $T$. Mahmood; investigation Noof T. Mahmood; resources, Noof T. Mahmood; data curation Noof T. Mahmood; Writing - original draft preparation Noof T. Mahmood; Writing - review and editing, Mahmuod H. Al-Muifraje and Sameer K. Salih; 
visualization, Sameer K. Salih; supervision Mahmuod H. Al-Muifraje; project administration, Mahmuod H. Al-Muifraje; funding acquisition, Noof T. Mahmood".

\section{Acknowledgement}

We would like to express our sincere to Alhikma collage university for technical support.

\section{References}

[1] X. Yang, X. Sun, D. Zhou, Y. Li, and H. Liu, "Towards wearable A-mode ultrasound sensing for real-time finger motion recognition", IEEE Transactions on Neural Systems and Rehabilitation Engineering, Vol. 26, No. 6, pp. 1199-1208, 2018.

[2] V. P. Maurya, P. Kumar, and S. Halder, Optimisation and Classification of EMG signal using PSO-ANN", Devices for Integrated Circuit (DevIC), pp. 191-195, 2019.

[3] P. Mccool, G. D. Fraser, A. D. Chan, L. Petropoulakis, and J. J. Soraghan, "Identification of contaminant type in surface electromyography (EMG) signals", IEEE Transactions on Neural Systems and Rehabilitation Engineering, Vol. 22, No. 4, pp. 774-783, 2014.

[4] S. M. Rissanen, M. Kankaanpää, M. P. Tarvainen, J. Nuutinen, I. Tarkka, A. Meigal, and P. A. Karjalainen, "Extraction of typical features from surface EMG signals in Parkinson's disease", In: Proc. of 11th International Congress of Parkinson's Disease and Movement Disorders, 2007.

[5] L. Balagourouchetty, J. K. Pragatheeswaran, B. Pottakkat, and G. Ramkumar, "GoogLeNetBased Ensemble FCNet Classifier for Focal Liver Lesion Diagnosis", IEEE Journal of Biomedical and Health Informatics, Vol. 24, No. 6, pp. 1686-1694, 2019.

[6] X. Yao, X. Wang, Y. Karaca, J. Xie, and S. Wang, "Glomerulus Classification via an Improved GoogLeNet", IEEE Access, Vol. 8, pp. 176916-176923, 2020.

[7] T. T. Hasan and A. H. Issa, "Human Facial Aggressive Detection System Based on FacialWidth-to-Height Ratio", IOP Conference Series: Materials Science and Engineering, Vol. 978, No. 1, pp. 012046, 2020.

[8] N. Nazmi, M. A. A. Rahman, S. I. Yamamoto, S. A. Ahmad, H. Zamzuri, and S. A. Mazlan, "A review of classification techniques of EMG signals during isotonic and isometric contractions", Sensors, Vol. 16, No. 8, pp. 1304,
2016.

[9] M. U. Khan, S. Aziz, M. Bilal, and M. B. Aamir, "Classification of EMG Signals for Assessment of Neuromuscular Disorder using Empirical Mode Decomposition and Logistic Regression", In: Proc. of International Conference on Applied and Engineering Mathematics (ICAEM), pp. 237-243, 2019.

[10] N. Amancherla, A. Turlapaty, and B. Gokaraju, "SVM based Classification Of sEMG Signals using Time Domain Features for the Applications towards Arm Exoskeletons", In: Proc. of the Advances in Robotics, pp. 1-5, 2019.

[11] M. Barsotti, S. Dupan, I. Vujaklija, S. Došen, A. Frisoli, and D. Farina, "Online finger control using high-density EMG and minimal training data for robotic applications", IEEE Robotics and Automation Letters, Vol. 4, No. 2, pp. 217223, 2018.

[12] L. S. Praveen, S. N. Nagananda, and P. Shankapal, "Design and Development of Real Time Bionic Hand Control Using EMG Signal", In: Proc. of IEEE International Conference on Electronics, Computing and Communication Technologies (CONECCT), pp. 1-4, 2018.

[13] T. T. Hasan, M. H. Jasim, and I. A. Hashim, "FPGA Design and Hardware Implementation of Heart Disease Diagnosis System Based on NVG-RAM Classifier", In: Proc. of Third Scientific Conference of Electrical Engineering (SCEE), pp. 33-38, 2018.

[14] Y. Narayan, R. M. Singh, L. Mathew, and S. Chatterji, "Surface EMG Signal Classification Using Ensemble Algorithm, PCA and DWT for Robot Control", In: Proc. of International Conference on Advanced Informatics for Computing Research, pp. 218-230, 2018.

[15] M. F. I. Ibrahim and A. A. A. Jumaily, "PCA indexing based feature learning and feature selection", In: Proc. of 8th Cairo International Biomedical Engineering Conference (CIBEC), pp. 68-71, 2016.

[16] S. R. Kurniawan and D. Pamungkas, "MYO Armband sensors and Neural Network Algorithm for Controlling Hand Robot", In: Proc. of International Conference on Applied Engineering (ICAE), pp. 1-6. 2018.

[17] W. Caesarendra, S. U. Lekson, K. A. Mustaqim, A. R. Winoto, and A. Widyotriatmo, "A classification method of hand EMG signals based on principal component analysis and artificial neural network", In: Proc. of International Conference on Instrumentation, Control and Automation (ICA), pp. 22-27. 2016.

[18] A. Dwivedi, Y. Kwon, A. J. McDaid, and M. 
Liarokapis, "A learning scheme for emg based decoding of dexterous, in-hand manipulation motions", IEEE Transactions on Neural Systems and Rehabilitation Engineering, Vol. 27, No. 10, pp. 2205-2215, 2019.

[19] H. Abdi and L. J. Williams, "Principal component analysis", Wiley interdisciplinary reviews: computational statistics, Vol. 2, No. 4, pp. 433-459, 2010.

[20] Ryu, Jaehwan, and D. H. Kim, "Real-time gait subphase detection using an EMG signal graph matching (ESGM) algorithm based on EMG signals", Expert Systems with Applications 85 (2017): 357-365.

[21] M. Awad and R. Khanna, "Support vector machines for classification", Efficient Learning Machines, pp. 39-66, 2015.

[22] G. Guo, H. Wang, D. Bell, Y. Bi, and K.Greer, "KNN model-based approach in classification", In: Proc. of OTM Confederated International Conferences on the Move to Meaningful Internet Systems", pp. 986-996, 2003.

[23] M. Z. Jahromi, E. Parvinnia, and R. John, "A method of learning weighted similarity function to improve the performance of nearest neighbor", Information sciences, Vol. 179, No. 17, pp. 2964-2973.2009.

[24] H. Dubey, "Efficient and accurate kNN based classification and regression", Center for Data Engineering, International Institute of Information Technology, Hyderabad-500, Vol. 32, 2013.

[25] A. Maghari, "Prediction of Student's Performance Using Modified KNN Classifiers", In: Proc. of the First International Conference on Engineering and Future Technology, pp. 143-150, 2018.

[26] G. Jia, H. K. Lam, S. Ma, Z. Yang, Y. Xu, and B. Xiao, "Classification of Electromyographic Hand Gesture Signals Using Modified Fuzzy CMeans Clustering and Two-Step Machine Learning Approach", IEEE Transactions on Neural Systems and Rehabilitation Engineering, Vol. 28, No. 6, pp. 1428-1435, 2020.

[27] P. Panday, N. Godara, "Decision support system for cardiovascular heart disease diagnosis using improved multilayer perceptron", International Journal of Computer Applications, Vol. 45, No. 8, 2012.

[28] P. Mccool, G. D. Fraser, A. D. Chan, A. L. Petropoulakis, and J. J. Soraghan, "Identification of contaminant type in surface electromyography (EMG) signals", IEEE Transactions on Neural Systems and Rehabilitation Engineering, Vol. 22, No. 4, pp.
774-783, 2014.

[29] Y. Paul, V. Goyal, and R. A. Jaswal, "Comparative analysis between SVM \& KNN classifier for EMG signal classification on elementary time domain features", In: Proc. of 4th International Conference on Signal Processing, Computing and Control (ISPCC), pp. 169-175. 2017. 